\title{
LOCAL VALUE DISTRIBUTION OF FUNCTIONS BOUNDED IN A HALF-PLANE
}

\author{
W. K. HAYMAN
}

\section{Introduction}

Suppose that $f(z)$ is meromorphic in an angle, which we may for definiteness take to be the right half-plane

$$
P:-\frac{\pi}{2}<\arg z<\frac{\pi}{2},
$$

and smooth at the origin, so that roots of $f=a$ do not accummulate there. In a recent paper [2] the notion of the inner order $k_{i}(P, f)$ of $f(z)$ in $P$ was introduced and so was the inner order $k_{i}(a, P, f)$ of the roots of the equation $f(z)=a$. One then obtains the following result, using ideas of Valiron [7].

Theorem A. We have $0 \leqq k_{i}(P) \leqq \infty$.

(i) If $1 \leqq k_{i}(P) \leqq \infty$, then $k_{i}(a, P)=k_{i}(P)$ except for at most two values a for which $k_{i}(a, P)<k_{i}(P)$.

(ii) If $0 \leqq k_{i}(P)<1$, then we have at most two values a for which $k_{i}(a, P)<k_{i}(P)$ and a certain small exceptional set $V$ of values a for which

$$
k_{i}(P)<k_{i}(a, P) \leqq 1 .
$$

It turns out that the nature of the set $V$ can be precisely described in terms of a set function due to Hyllengren [3, 4]. The positive theorems were obtained in [2] and examples are given in [1] for any value of the order $\varrho=k_{i}(P)$ satisfying $0 \leqq \varrho \leqq 1$.

If $f(z)$ is regular and bounded in $P$ then $f(z)$ does not assume large values $a$ and so $k_{i}(a, P)=0$ for such $a$. The small set $V$ in (ii) can certainly not contain all values in a disk and so we deduce that $k_{i}(P)=0$ for bounded functions. Thus we obtain results concerning the nature of $V$ for bounded functions by applying Theorem A with $k_{i}(P)=0$. In this paper we show, using a result on interpolation due to Katsnel'son [5] and Carleson (see appendix) that the results obtained in this way are sharp. The technique used by Drasin and the author [1] to obtain functions of order zero does not appear able to yield bounded functions although it does yield func- 
tions which are regular in $P$ and grow at most like $|z|$ as $z \rightarrow \infty$ in $P$. In order to obtain the result of Theorem 1 I needed an interpolation theorem such as Lemma 1. I wrote to Carleson about this and the theorem proved by him in the appendix was the result.

\section{Statement of results}

Let $V$ be a plane set and write

$$
e(x)=\exp \{-\exp \exp x\}
$$

Suppose that for some sequence $a_{n}$ of complex numbers and some positive $c$, every point of $V$ lies in infinitely many of the disks

$$
\left|a-a_{n}\right|<e(c n) \text {. }
$$

Then we say following Hyllengren [3,4] that the sequence $e(c n)$ majorises $V$. The span $s(V)$ of $V$ is defined to be the greatest lower bound of all numbers $c^{-1}$ for which $e(c n)$ majorises $V$. If $e(c n)$ does not majorise $V$ for any $c$, we say that $s(V)=\infty$. If $V=\cup V_{n}$, where $s\left(V_{n}\right)<\infty$ for each $n$, we say that $V$ has at most countably infinite span.

With the above definition the results on $V$ in [2] can be stated as follows.

Theorem B. If $\varrho=k_{i}(P)=0$ and in particular if $f(z)$ is regular and bounded in $P$, suppose that $0<\varrho^{\prime}<1$. Then if $V\left(\varrho^{\prime}\right)$ is the set of all a for which $k_{i}(a, P) \geqq \varrho^{\prime}$, we have

$$
s\left\{V\left(\varrho^{\prime}\right)\right\} \leqq\left\{\log \frac{1+\varrho^{\prime}}{1-\varrho^{\prime}}\right\}^{-1}
$$

Corollary. If $V(0)$ is the set of a for which $k_{i}(a, P)>0$ then $V(0)$ has at most countably infinite span. If $V(1)$ is the set for which $k_{i}(a, P)=1$, then

$$
s\{V(1)\}=0 .
$$

In this paper we obtain a converse result, at least if strict inequality holds in (2.3).

Theorem 1. Suppose that $0<\varrho^{\prime}<1$ and that $V^{\prime}=V\left(\varrho^{\prime}\right)$ is any bounded plane set such that

$$
s\left(V^{\prime}\right)<\left\{\log \frac{1+\varrho^{\prime}}{1-\varrho^{\prime}}\right\}^{-1}
$$

Then there exists $f(z)$ regular and bounded in $P$ and such that

for every $a \in V^{\prime}$.

$$
k_{i}(a, P) \geqq \varrho^{\prime}
$$


To define $k_{i}(a, P)$ we let $n_{\varepsilon}(r, a)$ be the number of roots of $f(z)=a$ in

$$
|z|<r,|\arg z|<\frac{\pi}{2}-\varepsilon
$$

and write

$$
k(a, \varepsilon)=\varlimsup_{r \rightarrow \infty} \frac{\log n_{\varepsilon}(r, a)}{\log r} .
$$

Then

$$
k_{i}(a, P)=\lim _{\varepsilon \rightarrow 0} k(a, \varepsilon)
$$

\section{The fundamental interpolation lemma}

We shall need to use the following result which is a very special case of Katsnel'son's sufficient condition. A more complete result is proved in the appendix.

Lemma 1. Suppose that $p_{n}$ is a sequence of positive integers, $a_{n}$ is a sequence of complex numbers, such that $\left|a_{n}\right| \leqq 1$, and $r_{n}$ is a sequence of positive numbers such that, for some positive constant $K$,

$$
\frac{r_{n+1}}{r_{n}} \geqq 1+K p_{n} p_{n+1}, \quad n=1,2, \ldots
$$

Then there exists a function $f(z)$ regular in $P$ and bounded there by a constant $K_{1}$ depending only on $K$, such that

$$
f\left(r_{n}\right)=a_{n}, f^{(p)}\left(r_{n}\right)=0, \quad 0<p \leqq p_{n}-1 .
$$

We write

$$
B_{n}(z)=\prod_{m \neq n}\left(\frac{r_{m}-z}{r_{m}+z}\right)^{p_{m}} .
$$

Katsnel'son [5] shows that, if

$$
\left|B_{n}\left(r_{n}\right)\right| \geqq \delta>0, \quad n=1,2, \ldots,
$$

then the interpolation problem (3.2) can be solved by a function $f(z)$ regular in $P$ and bounded by a constant depending on $\delta$ only. Thus to prove Lemma 1 we need only show that (3.1) implies (3.4). We proceed to prove this result. We denote by $K_{2}, K_{3}, \ldots$ positive constants depending on $K_{1}$ only.

We note first that (3.1) implies

$$
\frac{r_{n}}{r_{m}}>K_{2}(1+K)^{n-m} p_{m} p_{n}, \quad m<n
$$

If $n=m+1$ this follows from (3.1) with $K_{2}=K /(1+K)$. Next, if $n-m \geqq 2$, (3.1) 
shows that

Thus

$$
\frac{r_{v+1}}{r_{v}} \geqq 1+K, \quad n+1 \leqq v \leqq m-1 .
$$

and

$$
\frac{r_{m+1}}{r_{m}} \geqq K p_{m}, \quad \frac{r_{n}}{r_{n-1}} \geqq K p_{n}
$$

$$
\frac{r_{n-1}}{r_{m+1}} \geqq(1+K)^{n-m-2} .
$$

On multiplying these inequalities we obtain (3.5) with

$$
K_{2}=\left(\frac{K}{1+K}\right)^{2} .
$$

We now form the Blaschke products (3.3). We deduce from (3.5) that these products converge and in fact (3.5) yields

$$
\begin{gathered}
-\log B_{n}\left(r_{n}\right)<K_{3}\left\{\sum_{m<n} p_{m} \frac{r_{m}}{r_{n}}+\sum_{m>n} p_{m} \frac{r_{n}}{r_{m}}\right\} \\
\leqq \\
\sum_{m<n} \frac{K_{3}}{K_{2}}(1+K)^{m-n}+\sum_{m>n} \frac{K_{3}}{K_{2}}(1+K)^{n-m}=K_{4} .
\end{gathered}
$$

This proves (3.4) and thus Lemma 1 is proved.

We also need a form of the Milloux-Schmidt inequality.

Lemma 2. Suppose that $\varepsilon, \eta, r$ lie between 0 and 1 , that $F(z)$ is regular in $|z|<1$ and satisfies $|F(z)|<1$ there and further that

$$
\inf _{|z|=\varrho}|F(z)| \leqq \eta, \quad 0<\varrho<\varepsilon .
$$

Then for $\varepsilon / 2<r<1$, we have

$$
\log |F(z)|<\frac{\log \eta \log r}{6 \log (\varepsilon / 2)}, \quad|z|=r .
$$

We consider first the case $r=\varepsilon / 2$. Then since $|f(z)|<1$ in $|z|<\varepsilon$ and (3.6) holds, the classical Milloux-Schmidt inequality [6, Theorem 1, p. 107] yields for $|z|=r$

$$
\log |F(z)| \leqq(\log \eta)\left(1-\frac{4}{\pi} \tan ^{-1} \sqrt{1 / 2}\right)<\frac{1}{6} \log \eta
$$

Now Hadamard's convexity theorem shows that for $\varepsilon / 2 \leqq r<1$, we have

which is (3.7).

$$
\log |F(z)| \leqq \frac{\log (1 / r)}{\log (2 / \varepsilon)} \frac{1}{6} \log \eta,
$$

It is convenient to express this result a little differently. 
Lemma 3. Suppose that $F(z)$ is regular in $|z|<1$ and satisfies $|F(z)|<M$ there and further $|F(0)| \leqq 1$ and $|F(r)|=2$ where $1 / 2<r<1$. Then if $0<\varepsilon<1 / 2$, there exists $\varrho$, such that $0<\varrho<\varepsilon$ and

$$
|F(z)-F(0)| \geqq d,|z|=\varrho
$$

where

$$
\log \frac{1}{d}=\frac{6 \log (2 / \varepsilon) \log (M+1)}{\log (1 / r)}
$$

In particular if $F(z)-F(0)$ has a zero of order $p$ at the origin then the equation $F(z)=a$ has at least $p$ roots in $|z|<\varepsilon$, if $|a-F(0)|<d$.

We consider

$$
G(z)=\frac{F(z)-F(0)}{M+1}
$$

so that $|G(z)|<1$ for $|z|<1$. Suppose that (3.8) is false for $0<\varrho<\varepsilon$. Then we can apply Lemma 2 to $G(z)$ with

$$
\eta=\frac{d}{M+1}
$$

We obtain

$$
\log \left(\frac{1}{M+1}\right) \leqq \log |G(r)| \leqq \frac{\log (d /(M+1)) \log r}{6(\log (\varepsilon / 2))}
$$

i.e.

so that

$$
\log \left(\frac{M+1}{d}\right) \leqq \frac{6 \log (2 / \varepsilon)}{\log (1 / r)} \log (M+1)
$$

$$
\log \frac{1}{d}<\frac{6(\log (2 / \varepsilon)) \log (M+1)}{\log (1 / r)}
$$

Thus when (3.9) holds, (3.8) must be true for some $\varrho$. The last part of Lemma 3 follows at once from Rouché's theorem.

\section{A general example}

We can prove

Theorem 2. Suppose that $p_{n}, r_{n}$ satisfy the hypotheses of Lemma 1 and that $a_{n}$ is an arbitrary sequence of complex numbers satisfying $\left|a_{n}\right| \leqq 1$. Suppose further that $0<\varepsilon<1 / 2$. Then there exists $f(z)$ bounded and regular in $P$ and such that for 
$\left|a-a_{n}\right|<d_{n}$, the equation $f(z)=a$ has at least $p_{n}$ roots in $\left|z-r_{n}\right|<\varepsilon r_{n}$ where

$$
d_{n}=\exp \left(-K_{5} p_{n}\right)
$$

and $K_{5}$ is a constant depending on $\varepsilon$ and $K$ only.

We write $C=(1+K)^{1 / 2}-1$ and define $r_{n}^{\prime}$ by

We note that

$$
\frac{r_{n}^{\prime}}{r_{n}}=1+C p_{n} .
$$

$$
\begin{gathered}
\left(1+C p_{n}\right)\left(1+C p_{n+1}\right)=1+C\left(p_{n}+p_{n+1}\right)+C^{2} p_{n} p_{n+1} \\
\leqq 1+p_{n} p_{n+1}\left(2 C+C^{2}\right)=1+K p_{n} p_{n+1} .
\end{gathered}
$$

Thus

$$
r_{n+1} / r_{n}^{\prime} \geqq 1+C p_{n+1} .
$$

Hence the sequence $r_{1}, r_{1}^{\prime}, r_{2}, r_{2}^{\prime}, \ldots$ and the associated sequence $p_{1}, 1, p_{2}, 1, \ldots$ satisfies (3.1) with $C$ instead of $K$, and so we can find $f(z)$ satisfying the conditions of Lemma 1 , and in addition

$$
f\left(r_{n}^{\prime}\right)=2, \quad 1 \leqq n<+\infty .
$$

We now assume that $a_{n}=f\left(r_{n}\right)$ is a preassigned sequence such that $\left|a_{n}\right| \leqq 1$, and that

Consider

$$
|f(z)|<M .
$$

$$
F(\zeta)=f\left(r_{n} \frac{1+\zeta}{1-\zeta}\right)
$$

Then $F(\zeta)$ is regular in $|\zeta|<1,|F(\zeta)|<M$ there and $F(\zeta)-a_{n}$ has a zero order at least $p_{n}$ at the origin. Also

where $r$ is given by

$$
F(r)=2
$$

$$
r_{n} \frac{1+r}{1-r}=r_{n}^{\prime}, \quad \text { i.e. } \quad r=\frac{r_{n}^{\prime}-r_{n}}{r_{n}^{\prime}+r_{n}}=\frac{C p_{n}}{2+C p_{n}} .
$$

It now follows from Lemma 3 that $F(\zeta)$ assumes at least $p_{n}$ times in $|\zeta|<\varepsilon$ every value $a$, such that $\left|a-a_{n}\right|<d_{n}$ where

Thus

$$
\log \frac{1}{d_{n}}=\frac{6 \log (2 / \varepsilon) \log (M+1)}{\log \left(\left(2+C p_{n}\right) /\left(C p_{n}\right)\right)} .
$$

$$
\log \frac{1}{d_{n}} \leqq K_{5} p_{n}, \quad d_{n} \geqq \exp \left(-K_{5} p_{n}\right),
$$


where $K_{5}$ depends only on $K_{1}, M$ and $\varepsilon$ and so on $K_{1}$ and $\varepsilon$. Also if

$$
z=r_{n} \frac{1+\zeta}{1-\zeta}
$$

we have

$$
\left|z-r_{n}\right|=r_{n}\left|\frac{2 \zeta}{1-\zeta}\right|<4 \varepsilon r_{n}, \quad \text { if } \quad|\zeta|<\varepsilon<\frac{1}{2} .
$$

Thus the function $f(z)$ assumes every value $a$ such that $\left|a-a_{n}\right|<d_{n}$ at least $p_{n}$ times in $\left|z-r_{n}\right|<4 \varepsilon r_{n}$ and replacing $4 \varepsilon$ by $\varepsilon$ we deduce Theorem 2 .

\section{Proof of Theorem 1}

We now choose $\alpha=\varrho^{\prime}$, so that $0<\alpha<1$, set $c=\log ((1+\alpha) /(1-\alpha))$, and

$$
r_{n}=\exp \exp (c n), p_{n}=\left[2 r_{n}^{\alpha}\right]+1, \quad n \geqq 1,
$$

where $[x]$ denotes the integral part of $x$. Then

and so

$$
r_{n+1}=r_{n}^{(1+\alpha) /(1-\alpha)}
$$

$$
r_{n+1} / r_{n}=r_{n+1}^{\alpha} r_{n}^{\alpha} \geqq \max \left\{r_{1}^{2 \alpha}, \frac{p_{n} p_{n+1}}{9}\right\}
$$

Thus the conditions of Theorem 2 are satisfied, and taking $\varepsilon \leqq 1 / 2$ we see that $f(z)$ assumes the value $a$ at least $p_{n}$ times in $\left|z-r_{n}\right|<r_{n} \tan \varepsilon$ provided that

$$
\left|a-a_{n}\right|<d_{n} .
$$

If $a$ lies in infinitely many of the disks (5.2), then we see that the equation $f(z)=a$ has more than $\left(2 r_{n}\right)^{\alpha}$ roots in $|\arg z|<\varepsilon,|z|<2 r_{n}$ for infinitely many $n$. This implies by (2.4) and (2.5) that $k_{i}(a, P, f) \geqq \alpha$. Also the set of $a$ in question includes all $a$ lying in infinitely many of the disks (5.2). For $a_{n}$ we can choose any sequence such that $\left|a_{n}\right|<1$, and so any bounded sequence, for if $\left|a_{n}\right|<M$, where $M>1$, we consider $f / M, a_{n} / M$ instead of $f, a_{n}$. For $d_{n}$ we have from Theorem 2 and (5.1)

$$
d_{n}>\exp \left\{-3 K_{5} \exp (\alpha \exp (c n))\right\}>\exp \{-\exp \exp (c n)\}
$$

for large $n$, since $\alpha<1$. Thus for our exceptional set we can choose any bounded set $V^{\prime}$ of span less than $c^{-1}=\left\{\log \left(\left(1+\varrho^{\prime}\right) /\left(1-\varrho^{\prime}\right)\right)\right\}^{-1}$.

In conclusion we note that by using Theorem 2 and the technique employed in [1, Section 10] we can also deal with the limiting cases $\varrho^{\prime}=0$ and $\varrho^{\prime}=1$. In this way we can construct a regular bounded function in $P$, which assumes all values $a$ of a preassigned set of countably infinite span $V(0)$ with positive order $\varrho^{\prime}(a)$ and all vilues of a preassigned set $V(1)$ of zero span with order 1 . 


\title{
References
}

[1] Drasin, D., and W. K. HaYman: Value distribution of functions meromorphic in an angle. Proc. London Math. Soc. (3) (to appear).

[2] HaYman, W. K.: Value distribution and exceptional sets. - Lectures on approximation and value distribution, Sem. Math. Sup. 79. Presses Univ. Montréal, Montréal, Que., 1982, 79-147.

[3] Hyllengren, A.: Über die untere Ordnung der ganzen Funktion $f(z) e^{a z}$. - Festschrift zur Gedächtnisfeier für Karl Weierstraß 1815-1965, herausgegeben von H. Behnke und K. Kopfermann, Westdeutscher Verlag, Köln-Opladen, 1966, 555-577.

[4] Hyllengren, A.: Connections between two set functions. - Ark. Mat. 13, 1975, 273-285.

[5] KATSNel'Son, V. Ė.: Conditions for a system of root vectors of certain classes of operators to be a basis. - Funkcional. Anal. i Priložen. 1, 1967, 39-51 (Russian).

[6] Nevanlinna, R.: Analytic functions. - Springer-Verlag, Berlin-Heidelberg-New York, 1970.

[7] Valiron, G.: Directions de Borel des fonctions méromorphes. - Mémor. Sci. Math. 89, 1938, $1-70$.

\author{
Imperial College \\ Department of Mathematics \\ London SW7 2BZ \\ England
}

Received 6 October 1983 